\title{
Correction of Sturm-Liouville Eigenvalue Estimates
}

\author{
By J. Paine
}

\begin{abstract}
The error in the Sturm-Liouville eigenvalue estimates obtained by replacing the coefficient function with a piecewise constant interpolate is not uniform. In this paper we present a method for correcting these estimates to obtain a uniform approximation of all eigenvalues.
\end{abstract}

1. Introduction. The choice of numerical method for efficiently approximating a sequence of eigenvalues $\left\{\lambda_{k}\right\}_{k=1}^{m}$ of the regular Sturm-Liouville problem,

$$
\begin{aligned}
-\ddot{u}+q u=\lambda u, \quad u=u(x), \quad & \equiv \frac{d}{d x}, \quad x \in[0, \pi], \\
\alpha u(0)+\beta \dot{u}(0) & =0, \\
\gamma u(\pi)+\delta \dot{u}(\pi) & =0,
\end{aligned}
$$

depends on the desired accuracy of the estimates and also upon the number of eigenvalues needed. When a large number of uniformly accurate eigenvalue estimates are required, it is known [7] that standard methods such as [1], [4] or [6] encounter difficulties in efficiently estimating the higher eigenvalues because the corresponding eigenfunctions are highly oscillatory.

One method which has proved useful in approximating a sequence of eigenvalues (see, e.g., [2], [5], [8]) consists of choosing a partition $\Delta_{N}=\left\{0=x_{0}<x_{1}<\cdots<x_{N}\right.$ $=\pi\}$ of $[0, \pi]$ (usually uniform) and then finding the eigenvalues $\left\{\hat{\lambda}_{k}\right\}_{k=1}^{m}$ of the Sturm-Liouville problem

$$
\begin{gathered}
-\ddot{u}+\hat{q} u=\lambda u, \quad x \in[0, \pi], \\
\alpha u(0)+\beta \dot{u}(0)=0, \\
\gamma u(\pi)+\delta \dot{u}(\pi)=0,
\end{gathered}
$$

obtained from (1.1)-(1.3) by replacing $q$ by a piecewise polynomial interpolate $\hat{q}$ on $\Delta_{N}$.

The order of convergence of this method has been analyzed in [5] for piecewise constant interpolation and in [8] for general polynomial interpolation. The behavior of the error as a function of $k$ has been analyzed in [7], where it was shown that, when $\Delta_{N}$ is uniform and $\hat{q}$ is obtained using midpoint interpolation, the eigenvalue estimates satisfy

$$
\left|\lambda_{k}-\hat{\lambda}_{k}\right| \leqslant C h^{2}, \quad k=1,2, \ldots,[N / 2],
$$

where $h=\pi / N$ and $C$ is a constant bounded independently of $k$ and $h$. For values

Received April 7, 1981; revised December 14, 1981.

1980 Mathematics Subject Classification. Primary 65L15.

(C)1982 American Mathematical Society 0025-5718/82/0000-0339/\$03.00 
of $k$ greater than [N/2] there is a component of the error which grows rapidly as $k$ approaches multiples of $N$ and

$$
\left|\lambda_{k}-\hat{\lambda}_{k}\right| \leqslant C h, \quad k=1,2,3, \ldots,
$$

is the best bound that is valid for all values of $k$.

In this paper we present a simple modification of the above method which evaluates and removes the nonuniform component of the error in $\hat{\lambda}_{k}$ and hence yields uniform estimates of all eigenvalues.

2. Derivation of the Correction. We restrict attention to approximating problems of the form (1.4)-(1.6), where $\hat{q}$ is defined by

$$
\hat{q}(x)=q\left(x_{i+1 / 2}\right), \quad x \in\left[x_{i}, x_{i+1}\right], i=0,1, \ldots, N-1,
$$

on the uniform partition $\Delta_{N}$ of $[0, \pi]$ and $x_{i+1 / 2}=\frac{1}{2}\left(x_{i}+x_{i+1}\right)$. We further assume that the eigenfunction $\hat{u}_{k}$ of (1.4)-(1.6), corresponding to the eigenvalue $\hat{\lambda}_{k}$, is normalized so that

$$
\left\|\hat{u}_{k}\right\|_{2}=\left\|u_{k}\right\|_{2}=1 \text { and } \int_{0}^{\pi} u_{k} \hat{u}_{k} d x>0 .
$$

In order to derive the required correction factor and to establish the uniformity of the error in the corrected eigenvalue estimates, we firstly state some results derived in [7].

LEMMA 2.1. Let $q \in C^{2}[0, \pi], f \in C^{1}[0, \pi]$ and $\hat{q}$ be the midpoint interpolate of $q$ on the uniform partition $\Delta_{N}$ of $[0, \pi]$. Then

$$
\left|\int_{0}^{\pi}(q-\hat{q}) f d x\right| \leqslant \pi\left\{\frac{1}{4}\left\|q^{\prime}\right\|_{\infty}\left\|f^{\prime}\right\|_{\infty}+\frac{1}{8}\left\|q^{\prime \prime}\right\|_{\infty}\|f\|_{\infty}\right\} h^{2}
$$

LEMMA 2.2. Let the conditions of Lemma 2.1 be satisfied and define

$$
\rho=\frac{1}{2} \min _{k}\left|\lambda_{k+1}-\lambda_{k}\right| \text {. }
$$

Then

$$
\begin{aligned}
\left|\lambda_{k}-\hat{\lambda}_{k}-\int_{0}^{\pi}(q-\hat{q}) u_{k}^{2} d x\right| & \leqslant 4 \rho^{-1}\|q-\hat{q}\|_{\infty}^{2}, \quad k=1,2,3, \ldots, \\
& \leqslant \rho^{-1}\left\|q^{\prime}\right\|_{\infty}^{2},
\end{aligned}
$$

if $\|q-\hat{q}\|_{\infty}<\frac{1}{2} \rho$.

Lemma 2.2 indicates that there are two (not independent) principal components which comprise the error $\left|\lambda_{k}-\hat{\lambda}_{k}\right|$. The first, $4 \rho^{-1}\|q-\hat{q}\|_{\infty}^{2}$, is due to approximating $q$ with a piecewise constant interpolate and is independent of $k$. The second, $\left|\int_{0}^{\pi}(q-\hat{q}) u_{k}^{2} d x\right|$, is the error in evaluating $\int_{0}^{\pi} q u_{k}^{2} d x$ using product midpoint quadrature, and obviously depends on $k$. To examine this dependence we note [3, p. 336] that $u_{k}=v_{k}+O\left(k^{-1}\right)$, where $v_{k}=A_{k} \sin \left(\mu_{k} x+\phi_{k}\right)$ is the eigenfunction of (1.1)-(1.3) with $q \equiv 0$, corresponding to the eigenvalue $\mu_{k}$ and normalized in the same manner as $\hat{u}_{k}$. This asymptotic result used in conjunction with Lemma 2.1 gives

$$
\left|\int_{0}^{\pi}(q-\hat{q}) u_{k}^{2} d x\right| \leqslant\left|\int_{0}^{\pi}(q-\hat{q}) v_{k}^{2} d x\right|+C h^{2} .
$$


On noting that $\mu_{k}=k+O(1)$, a further application of Lemma 2.2 with $f=v_{k}^{2}$ yields

$$
\left|\int_{0}^{\pi}(q-\hat{q}) v_{k}^{2} d x\right| \leqslant C_{2} k h^{2}
$$

and hence, from (2.1) and (2.2),

$$
\left|\lambda_{k}-\hat{\lambda}_{k}\right| \leqslant C_{1} h^{2}+C_{2} k h^{2},
$$

where $C_{1}$ and $C_{2}$ depend only on $q$.

Examining this dependence on $q$ further, we see that if $q$ is a rapidly varying function, then the smallest value of $N$ which will make the component $4 \rho^{-1}\|q-\hat{q}\|_{\infty}^{2}$ sufficiently small to give the desired accuracy in the eigenvalue estimates will probably be large enough so that (1.7) will guarantee uniformity of the error for the eigenvalues required. However, if $q$ is only slowly varying or if a particularly long sequence of eigenvalues are required, then this value of $N$ may not be large enough in order to apply (1.7), and the growth exhibited in (2.3) may become apparent in the estimates.

In the latter case one could simply take $N$ to be at least twice the number of eigenvalues required, however, if we can (uniformly) estimate the component of the error which grows with $k$, then a potentially more efficient approach would be to correct the eigenvalues using this information and hence obtain uniform estimates.

Returning to (2.1), we see that the component of the error that grows with $k$ depends on $u_{k}$ (the exact eigenfunction) and so cannot be estimated directly. But if we use (2.2), (2.1) can be replaced by

$$
\left|\lambda_{k}-\hat{\lambda}_{k}-\int_{0}^{\pi}(q-\hat{q}) v_{k}^{2} d x\right| \leqslant C h^{2}, \quad k=1,2,3, \ldots .
$$

Since $q, \hat{q}$ and $v_{k}$ are known functions, we could now apply a standard quadrature rule to evaluate $\int_{0}^{\pi}(q-\hat{q}) v_{k}^{2} d x$, except that we would still face the problem of uniformly estimating the integral because of its dependence on $v_{k}$. However, if we note that

$$
\left|q(x)-q\left(x_{i+1 / 2}\right)-q_{h}(x)\right| \leqslant C h^{2}, \quad x \in\left[x_{i}, x_{i+1}\right],
$$

where $q_{h}(x)=h^{-1}\left(x-x_{i+1 / 2}\right)\left(q_{i+1}-q_{i}\right)$ and $q_{i}=q\left(x_{i}\right)$, then obviously $\left\|q-\hat{q}-q_{h}\right\|_{\infty} \leqslant C h^{2}$ and hence

$$
\begin{aligned}
\left|\int_{0}^{\pi}(q-\hat{q}) v_{k}^{2} d x\right| & \leqslant\left|\int_{0}^{\pi} q_{h} v_{k}^{2} d x\right|+\left|\int_{0}^{\pi}\left(q-\hat{q}-q_{h}\right) v_{k}^{2} d x\right| \\
& \leqslant\left|\int_{0}^{\pi} q_{h} v_{k}^{2} d x\right|+\left\|q-\hat{q}-q_{h}\right\|_{\infty}\left\|v_{k}\right\|_{2}^{2} \leqslant\left|\int_{0}^{\pi} q_{h} v_{k}^{2} d x\right|+C h^{2} .
\end{aligned}
$$

Since $q_{h}$ is a piecewise linear polynomial and $v_{k}$ is a trigonometric function, the integral $\int_{0}^{\pi} q_{h} v_{k}^{2} d x$ can now be evaluated exactly, and we have

$$
\begin{aligned}
\int_{0}^{\pi} q_{h} v_{k}^{2} d x & =\frac{1}{2} A_{k}^{2}\left\{\int_{0}^{\pi} q_{h} d x-\int_{0}^{\pi} q_{h} \cos 2\left(\mu_{k} x+\phi_{k}\right) d x\right\} \\
& =\frac{1}{2} h A_{k}^{2}\left\{\frac{\sin \gamma \mu_{k} h}{\mu_{k} h^{2}}-\frac{\cos \gamma \mu_{k} h}{\sqrt{ } \mu_{k} h}\right\} \sum_{i=0}^{N-1}\left(q_{i+1}-q_{i}\right) \sin 2\left(\mu_{k} x_{i+1 / 2}+\phi_{k}\right)
\end{aligned}
$$

since $\int_{0}^{\pi} q_{h} d x=0$. 
In summary then we have

THEOREM 2.1. Let the conditions of Lemmas 2.1 and 2.2 be satisfied. Then,

$$
\left|\lambda_{k}-\bar{\lambda}_{k}\right| \leqslant C h^{2}, \quad k=1,2,3, \ldots
$$

where

$$
\bar{\lambda}_{k}=\hat{\lambda}_{k}+\int_{0}^{\pi} q_{h} v_{k}^{2} d x
$$

and $C$ is bounded independently of $k$ and $h$.

Proof. This result follows directly from (2.4) and (2.5).

3. A Numerical Example. To illustrate the improvement of the eigenvalue estimates which can be obtained by using the corrected estimates (2.6), we calculated $\left\{\hat{\lambda}_{k}\right\}_{k=1}^{40}$ for the eigenvalue problem

$$
-\ddot{u}+e^{x} u=\lambda u, \quad x \in[0,1], u(0)=0=u(1),
$$

with $N=16$. The error in these estimates is plotted against $k$ in Figure 3.1 and clearly shows both the uniformity of the error for $k<N / 2$ and also the growth as $k$ approaches multiples of $N$. If we now use (2.6) to generate the corrected estimates, then the error, shown in Figure 3.2, is obviously superior to the error in the uncorrected estimates and can be seen to achieve the desired uniformity.

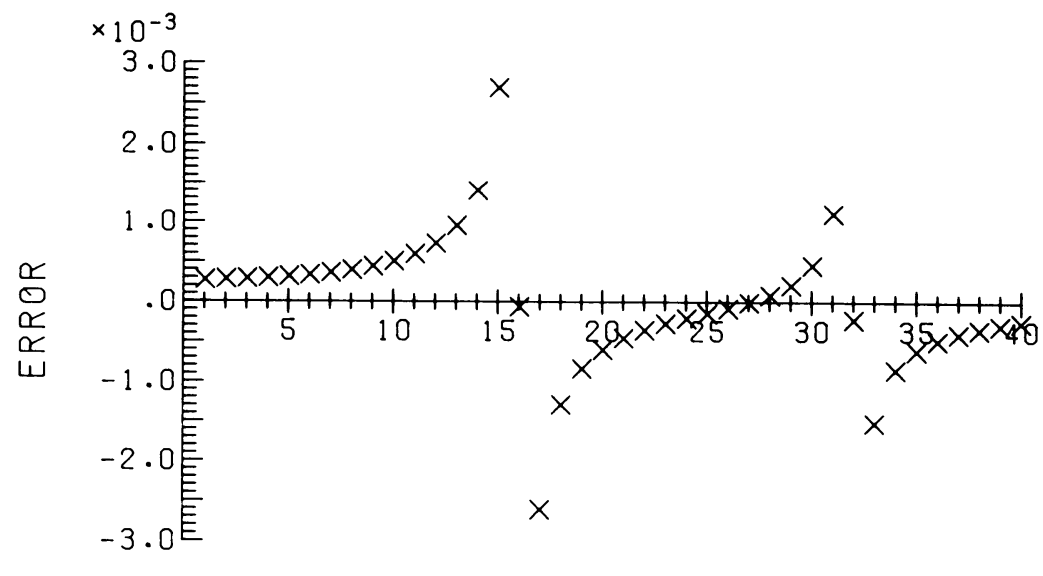

\section{E I GENVALUE}

FIGURE 3.1

Eigenvalue error for (3.1) obtained by approximating the differential equation

The reason for the anomalous behavior of the error for $k=16$ and 32 (i.e. multiples of $N$ ) is not clear, though it should be noted that this behavior is not in conflict with the bound given in Theorem 2.1. 


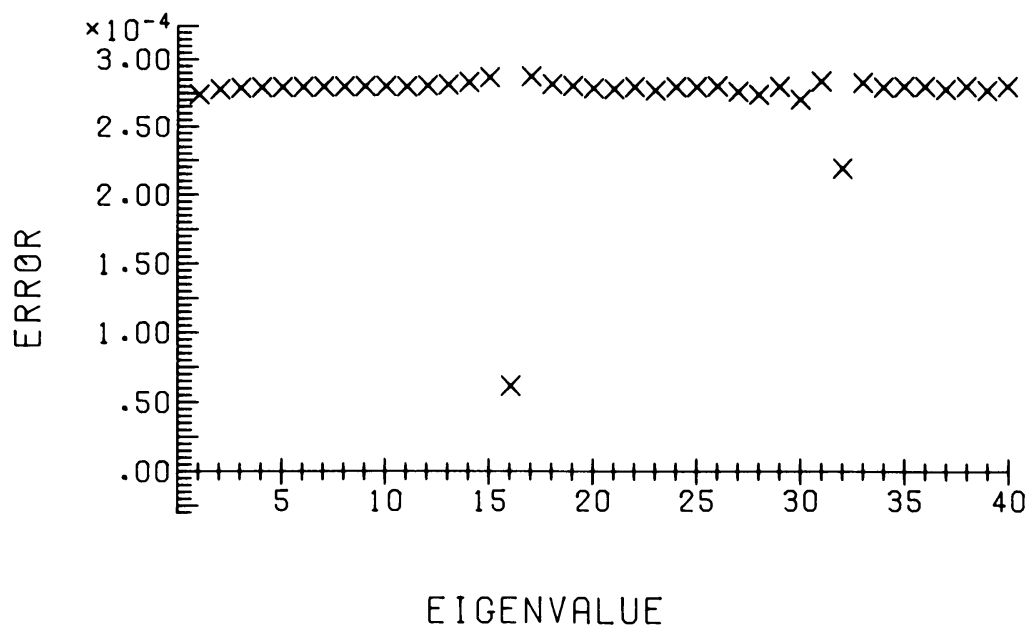

Figure 3.2

Eigenvalue error in using (2.6) to correct the eigenvalue estimates

4. Conclusion. In approximating Sturm-Liouville eigenvalues, the growth of the error with $k$ often reflects the increasingly oscillatory behavior of the eigenfunctions rather than any difficulty caused by the particular coefficient function. In this paper we have identified, and shown how to remove, this nonuniform component of the error for one particular method for approximating eigenvalues. Thus, for any given Sturm-Liouville eigenvalue problem, the difficulty in obtaining acceptable estimates of a sequence of eigenvalues using this corrected method will be solely due to the characteristics of the coefficient function rather than the number of eigenvalues required.

This method of correcting the eigenvalue estimates by evaluating and removing the dominant $k$-dependent component of the error should also be applicable to the more general methods given in [8], where $q$ is approximated by a piecewise polynomial of degree $n \geqslant 1$. Though in this case the remaining error terms will still depend on $k$, and hence the effect of the correction will be to reduce the rate of growth of the error rather than to eliminate it altogether.

Acknowledgements. The author wishes to express his gratitude to Frank de Hoog for suggesting this problem, and would also like to thank the referee for his constructive criticism of the first version of this paper.

\footnotetext{
Computer Science Department

University of Bristol

Bristol, England
}

1. G. Birkhoff, C. DE Boor, B. Swartz \& B. WendrofF, “Rayleigh-Ritz approximation by piecewise polynomials,” SIAM J. Numer. Anal., v. 3, 1966, pp. 188-203.

2. J. Canosa \& R. Gomes DE Oliveira, "A new method for the solution of the Schrödinger equation," J. Comput. Phys., v. 5, 1970, pp. 188-207.

3. R. Courant \& D. Hilbert, Methods of Mathematical Physics, Vol. I, Interscience, New York, 1953. 
4. B. HuBbARD, "Bounds for eigenvalues of the Sturm-Liouville problem by finite difference methods," Arch. Rational Mech. Anal., v. 10, 1962, pp. 171-179.

5. L. GR. IXARU, “The error analysis of the algebraic method for solving the Schrödinger equation," $J$. Comput. Phys., v. 9, 1972, pp. 159-163.

6. H. B. Keller, Numerical Methods for Two-point Boundary Value Problems, Blaisdell, Waltham, Mass., 1968.

7. J. W. PAINE \& F. R. DE HoOg, "Uniform estimation of the eigenvalues of Sturm-Liouville problems," J. Austal. Math. Soc. Ser. B, v. 21, 1980, pp. 356-383.

8. S. PRUESS, "Estimating the eigenvalues of Sturm-Liouville problems by approximating the differential equation," SIAM J. Numer. Anal., v. 10, 1973, pp. 55-68. 